\title{
Sensitivity to shifts of a point stimulus: An instance of tactile hyperacuity
}

\author{
JACK M. LOOMIS and CARTER C. COLLINS \\ University of California, Santa Barbara, California 93106 \\ and \\ Smith-Kettlewell Institute of Visual Sciences, Pacific Medical Center, San Francisco, California 94115

\begin{abstract}
The sensitivity of the tactile sense to shifts in the position of a point stimulus was
\end{abstract} \\ determined at four body loci: the index finger, forehead, belly, and back. Water-jet stim- \\ ulation was used, a method which allows frictionless travel of a point stimulus over the \\ skin. Three subjects were tested by means of the method of forced choice, with the \\ task being to say whether the stimulus moved to the right or to the left. The shift \\ threshold was defined as that shift excursion which gave rise to $75 \%$ correct responding. \\ The basic finding was that shift thresholds were on the order of 10 to 30 times smaller \\ than the corresponding two-point limens obtained by Weinstein (1968) for the same body loci. \\ These unexpectedly small shift thresholds indicate an exquisite sensitivity of the cutaneous \\ sense to rapid changes in position of a point stimulus. A physiological model is presented \\ which accounts for the difference between localization and spatial resolution.
}

It has long been known that the tactile sense is capable of localizing a point stimulus with considerably greater precision than it can resolve two points. In particular, the conventional "error of localization" is between one-fourth and one-third the two-point limen for virtually all body loci (Boring, 1930, 1942; Weinstein, 1968; Zigler, 1935). This substantial difference between the indices of spatial resolution and point localization has been a consistent finding even with variations in the experimental procedure, the subject's task demands, and the statistical definition used for each index (Zigler, 1935); as such, it must reflect a basic property of cutaneous processing. The present experiment was undertaken to determine the limits of the localizing capability of the skin under conditions which were deemed optimal. Specifically, a forcedchoice procedure was employed in a task requiring the subject to discriminate the shifts in position of a point stimulus. The results obtained are sufficient reason to characterize tactile sensitivity to shifts in location as a form of "hyperacuity," a label Westheimer (1977; Westheimer \& McKee, 1977a, 1977b) has applied to certain forms of visual spatial sensitivity.

\section{METHOD}

\footnotetext{
Apparatus

Well-defined stimuli were presented using a water-jet stimulator, which at the time was a precursor of a more elaborate stimulator capable of presenting complex patterns under the control of video input (Collins \& Madley, Note 1). Water-jet stimulation used in

This research was supported by Department of Health, Education, and Welfare Grant 14-P-SS282/9 from the Social and Rehabilitation Services, NIH Research Grant R01-EY-00686 from the National Eye Institute, and the Smith-Kettlewell Eye Research Foundation.
}

conjunction with a water-tight membrane has several properties to recommend it: (1) Its spatial, temporal, and intensive parameters can be controlled with a high degree of precision; (2) even for irregular skin surfaces, stimulus coupling is uniform; and, (3) travel of a point stimulus across the surface of the skin is completely frictionless. A disadvantage of this form of stimulation is that two or more water jets presented simultaneously and in close proximity interact to an extent that makes stimulation with multiple points infeasible.

The apparatus we used was constructed specifically for this experiment. In general terms, the system conducts water from the plumbing system of the building to a small nozzle, the flow of which is directed against a water-tight membrane; ancillary components permit lateral displacement of the water jet as well as control over its intensive and temporal parameters.

Water conduction was accomplished using plastic tubing fastened onto $1 / 4$-in. copper tubing. Valves and other fittings were standard copper plumbing fixtures with $1 / 4$-in inner diameters. The system was built over a laboratory sink and connected to its tap. Water pressure was monitored by a pressure gauge incorporated into the system, with any observed pressure changes being compensated by adjustment of the tap flow; pressure was maintained at 20 psi. A solenoid Y valve (Skinner Electric B-4 DA 1 075) run on $12 \mathrm{~V}$ dc and actuated by a microswitch was used to divert flow from a runoff water jet to the water jet used as the stimulator. The solenoid valve operates quickly, giving virtually instantaneous rise and fall of the water jet stimulus. Each water jet nozzle consisted of a straight hollow tube of constant width (1.3 mm i.d.) which was obtained by removing the tapered portion of a hypodermic needle. Because the two nozzles permitted the same volume flow $(13.8 \mathrm{cc} / \mathrm{sec}$ at $20 \mathrm{psi})$, no change was observed in the water pressure as the flow was diverted from one channel to the other.

The stimulating water jet was mounted on a microscope ștage positioner (with vernier scale), which could be moved in the horizontal direction transverse to that of the water jet. The jet was directed through the open side of a plastic containment box and onto the inner surface of a flexible rubber membrane mounted on the opposite side of the box over a window, 3 in. square; the membrane was a 4-in. square of dental dam material. The surface of the body to be tested was placed up against the outer surface of this membrane. The stage positioner, containment box, and other components of the system were all rigidly fixed in 
position by means of rods and clamps mounted onto a wooden base over the sink.

From the orifice of the nozzle, the jet of water traveled $15 \mathrm{~cm}$ to the surface of the membrane; in this distance, the jet increased in diameter from 1.3 to $1.8 \mathrm{~mm}$. We have no measurements of the indentation of the skin, but it undoubtedly was over $2 \mathrm{~mm}$ in diameter. Force measurements made with a strain gauge placed against the outer surface of the membrane indicated that the jet exerted a force of about $6.4 \times 10^{3}$ dynes $(6.5 \mathrm{~g})$.

\section{Subjects}

A complete set of data was collected for two subjects W.G. and J.L., the first author. A less complete set of data was obtained for a third subject, F.M., with additional data of a nonsystematic nature collected on three others. W.G. is male and blind, J.L. male and sighted, and F.M. female and sighted. All had extensive practice as psychophysical observers, although J.L. and F.M. had little prior exposure to this task. At the time of this experiment, the three subjects ranged in age from 25 to 30 .

\section{Body Loci}

Four body loci were tested. For testing of the distal pad of the right index finger, a horizontal tray was mounted beneath the containment box so that the subject could rest and steady his arm while holding his finger vertically against the rubber membrane. The other loci tested were the middle of the forehead, the belly near the midline just above the navel, and the back just to either side of the midline at a vertical position opposite to the position tested on the belly. For all body loci, the point stimulus moved left or right in a direction transverse to the longitudinal axis of the part tested. The forehead and finger were tested with the subject seated, while the other loci were tested with the subject standing. Subjects steadied themselves with their free hands during testing so that body sway would exert little effect on the data.

\section{Procedure}

In most cases, the subjects were tested on only a single body locus during any one session. The order of testing the various body loci was randomized. The method consisted of presenting a block of trials on each of which the water jet was translated a given distance to the left or right. The starting point was varied from trial to trial so that subjects could not use the absolute stimulus position as a cue but had to rely solely on the shift in location. After each shift, which was forwarned, the subject indicated whether the shift was to the right or left; he was then given feedback. Interspersed blocks of 50 trials each of various shift magnitudes were run during a session (two blocks of each magnitude). Shift magnitudes were selected with the intention of bracketing the $75 \%$ correct performance level. An ongoing account of response accuracy was maintained during the session so that, where necessary, additional shift values were tested to achieve this bracketing (all values tested being represented in the Results). Throughout testing, the subjects wore earphones connected to a white noise source which served to mask any possible auditory cues as the jet moved over the membrane.

The procedure on a given trial went as follows: The trial was initiated with the experimenter's actuating the solenoid valve that turned on the stimulating jet. About $1 \mathrm{sec}$ later, a "ready" signal was voiced by the experimenter, followed shortly by the experimenter's moving the jet to the right or left according to a random sequence. (With relatively little practice, the experimenter was able to move the stimulus the desired amount in one smooth motion while looking at the vernier scale through a magnifying glass.) The shift was accomplished usually in about .3 to $.5 \mathrm{sec}$, although the longer excursions ( 3 to $6 \mathrm{~mm}$ ) took up to perhaps $1 \mathrm{sec}$. About $1 \mathrm{sec}$ after the shift, the stimulus presentation ended as the jet was turned off. The subject terminated the trial with his response of "left" or "right."

\section{RESULTS}

Figure 1 gives the percentage of correct responses for each shift magnitude, body locus, and subject. Observe that for two of the subjects, the smallest measurable excursion of $.1 \mathrm{~mm}$ still allowed greater than $75 \%$ correct discrimination with the index finger. A fourth subject, also tested on the finger, gave similar results $(80 \%$ at $.1 \mathrm{~mm})$, implying that the sensitivity of the index finger to shifts in stimulus position is extraordinary even when the "point" stimulus is more than $2 \mathrm{~mm}$ in diameter.

For the definition of the shift threshold, we chose the shift excursion (interpolated) which gave rise to $75 \%$ correct responding; in the case of the index finger for J.L. and W.G., .1 mm was taken as a conservative estimate of the threshold. The resulting threshold values for each body locus were then averaged for subjects J.L. and W.G., since they were the subjects for whom complete data sets were obtained. These average values are plotted in Figure 2 along with the average twopoint discrimination thresholds and point localization thresholds determined for the same body loci by Weinstein (1968).

\section{DISCUSSION}

\section{Most of the early studies of tactile point localization}
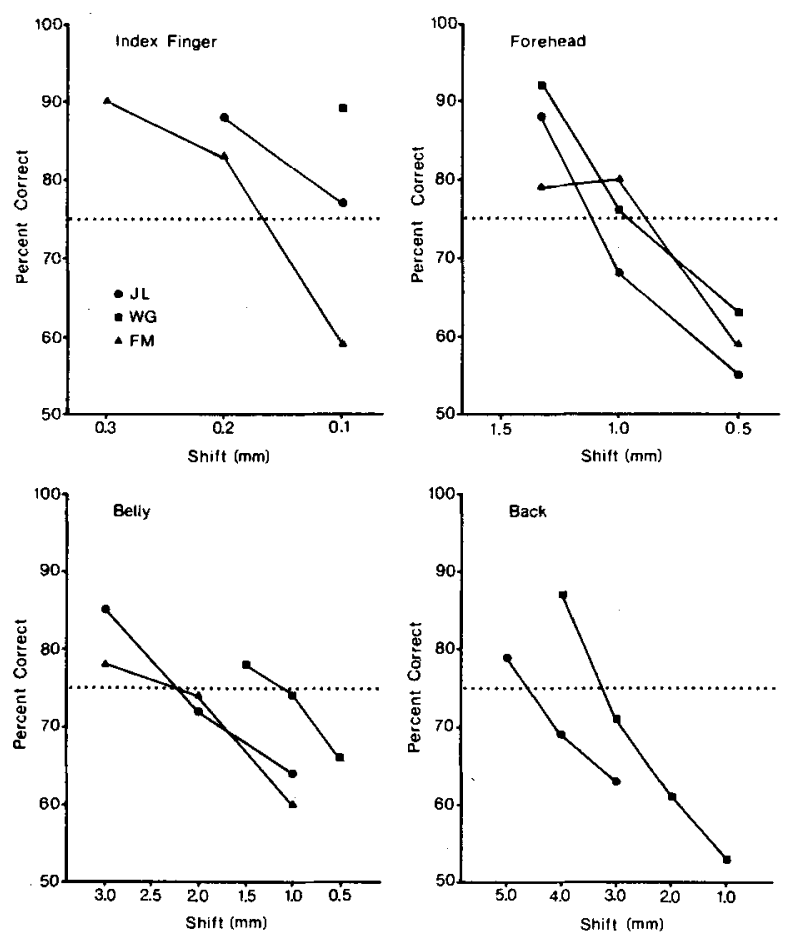

Figure 1. Percentage of correct responses as a function of shift excursion, body locus, and subject. 


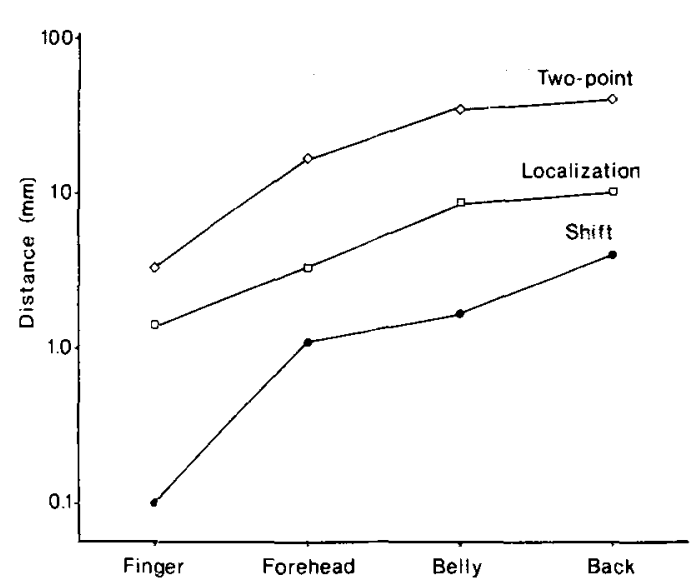

Figure 2. Cutaneous thresholds as a function of body locus. (The shift thresholds from the present study are plotted together with the two-point and point-localization thresholds obtained by Weinstein, 1968.)

(see Boring, 1942) used some variant of the following procedure: The experimenter touched the skin surface of the subject with a stylus and the subject then attempted to touch the same point unaided by vision. The error of localization was taken as the average absolute error over a series of trials. A slightly more refined procedure was employed by Weinstein (1968); in it, the experimenter touched the skin with a stylus first at a reference point and then at a second point some distance away. The subject's task was to say whether or not the second stimulus coincided with the reference point. The error of localization was defined as the separation between the reference point and the most distant point over a series of trials that was identified by the subject as the reference point. Given these comparatively crude procedures and ways of defining the error of localization, it comes as little surprise that the shift thresholds in the present study are smaller. What is surprising is the magnitude of the difference between the two measures. When the subject's task is simplified to one of discriminating left from right shifts, the resulting performance is quite remarkable. However, it may be incorrect to attribute the entire difference between the two indices to the reduction in the memorial and judgmental demands made on the subject and to the different statistical treatments. It may well be that the motion of the shifting stimulus brings into play additional mechanisms not activated by static presentation methods.

An issue of considerable theoretical interest concerns the consistent finding that localization acuity, as measured by the error of localization, is finer than spatial resolution, as measured by the two-point limen' (Boring, 1930, 1942; Weinstein, 1968; Zigler, 1935). The present study accentuates this contrast between point localization and resolution with its finding that shift thresholds are roughly 10 to 30 times finer than the corresponding two-point thresholds obtained by Weinstein (1968); furthermore, it is interesting that the .1-mm shift threshold for the finger pad is only a fracton of the 2-mm widths of the smaller receptive fields of mechanoreceptive units in the finger (Johansson, 1976).

It is the fineness of the shift thresholds which prompted the term "tactile hyperacuity" in analogy with Westheimer's use of "visual hyperacuity" in referring to the exquisite visual sensitivity observed with a variety of spatial discrimination tasks such as vernier alignment and spatial interval discrimination (Berry, 1948; Ludvigh, 1953; Westheimer, 1977; Westheimer \& McKee, 1977a, 1977b). However, in using the term, we do not mean to imply (nor does Westheimer) that there is anything paradoxical about these acuities exceeding spatial resolution. As is evident from consideration of the various hyperacuity configurations, the required spatial discriminations are made on the basis of pattern characteristics which are little affected by the spatial frequency filtering (optical or neural blurring) which limits spatial resolution. Regarding the present instance, Loomis (1974) has argued that localization of a point stimulus which has undergone low-pass spatial filtering is readily accomplished using the phase information in the spatial frequencies constituting the remaining pattern content. In support of this line of reasoning and as proof that the effect is not peculiar to the sense of touch, Apkarian-Stielau and Loomis (1975) showed that under conditions of optical blurring, visual discrimination of left from right shifts of a bar target was considerably better than resolution of two simultaneously presented targets. Although, with some input-output systems, localization (in time or in space) might be theoretically independent of resolution (temporal or spatial), this is clearly not the case with the sense of touch. As earlier work has shown (Boring, 1930; Weinstein, 1968) and the present work affirms, localization acuity covaries with spatial resolution, surely reflecting the innervation densities, receptive field sizes, and areas of cortex associated with different body loci (Johansson \& Vallbo, 1976; Weinstein, 1968; Werner \& Whitsel, 1973).

In physiological terms, how might we understand the disparity between localization and resolution? Interest in this question dates back to Weber (Boring, 1942; Sinclair, 1967), who speculated that each cutaneous nerve fiber innervated an area of the skin surface, which he termed a "sensory circle" (the current receptive field notion). He supposed that cutaneous sensibility at a particular body locus was subserved by a mosaic of densely packed nonoverlapping sensory circles (actually hexagons). In the two-point discrimination task the liminal sensation of duality was experienced when there was stimu- 
lation of two sensory circles separated by one which was quiescent. Localization, on the other hand, was limited by the spacing between centers of adjacent circles, because stimulation anywhere within one circle was experienced as a constant locus of stimulation.

In the ensuing years, there was considerable debate as to whether the same or different physiological mechanisms were involved in the two tasks (Boring, 1942; Sinclair, 1967, pp. 13, 179). At the one extreme, Rivers and Head (1908) claimed that two-point discrimination was mediated solely by the "epicritic sensibility" of the skin but that localization was subserved both by epicritic sensibility and by the "deep sensibility" of muscle afferents underlying the skin. At the other extreme, Boring (1930) argued that precisely the same mechanisms were involved, but that the difference between the two capabilities could be explained by a statistical version of Weber's sensory circle idea.

The model we are proposing is a compromise between these two extremes, for it utilizes the same physiological structures but different neural codes in accounting for the two types of sensitivity. Thus, in this model, two-point discrimination and point localization are two quite different capabilities, in contrast to the view expressed by Boring (1942, p. 483) that two-point discrimination is a special case of localization wherein one point is localized with respect to the other rather than with respect to the body schema.

The model we propose has as its precursors the speculations of Hoagland (1932), von Békésy (1967), and Zigler (1935). It is based on the following assumptions:

(1) Cutaneous sensibility at each location on the skin is mediated by a small number of mechanoreceptive units with overlapping receptive fields of nonuniform sensitivity (see Figure 3).

(2) The instantaneous response of each mechanoreceptive unit is a single-valued linear function of the effective stimulation within its receptive field. Effective stimulation at each point in the receptive field is the product of stimulus amplitude and sensitivity of the unit at that point.

(3) Localization of a point stimulus is signaled by the local signs of the stimulated units with "fine tuning" provided by their relative response magnitudes. With regard to the fine tuning, relational comparison of different units might be in terms of the ratios or differences of their responses. A physiological realization of this comparison might be accomplished by means of algebraic summation of excitation and inhibition at some succeeding level of the cutaneous pathway; this would be analogous to opponent-process recoding of receptor responses in the processing of color.

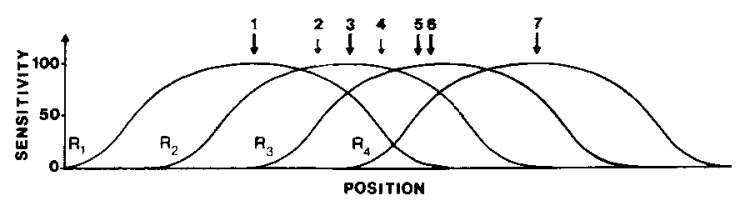

Figure 3. Schematic representation of the overlapping receptive fields of four mechanoreceptive units and seven point stimuli. (See text for discussion of the model.)

(4) The sensation of duality in the two-point discrimination task is signaled by three or more adjacent units: equality of responses in the outer units together with smaller responses in the intervening ones, with the decrement surpassing some criterion value [analogous to the Rayleigh criterion in optics (Hecht \& Zajac, 1976; Westheimer, 1972)].

To examine the behavior of such a model, we can represent the responses of the four mechanoreceptive units in Figure 3 (identified by their receptive fields $\left.R_{i}\right)$ as a vector $\left(r_{1}, r_{2}, r_{3}, r_{4}\right)$. Let us first consider point localization. If we use the computational scheme outlined above and assume that Stimuli 1, 3, 5, 6, and 7 (Figure 3) each have amplitudes of 1.0 , then the corresponding response vectors would be $(100,70,0,0),(70,100,70,0)$, $(7,84,98,51),(0,77,99,64)$, and $(0,0,70,100)$. If some relational comparison is made between the responses of adjacent units, it is evident that even Stimuli 5 and 6 can be discriminated with respect to location. Sensitivity to location depends very much upon the slopes of the underlying sensitivity functions. An interesting finding regarding point localization is that it depends only weakly, if at all, on stimulus amplitude (Disher, 1933; Pritchard, 1931); moreover, a point stimulus which is detectable is also localizable (Pritchard, 1931). We might account for this invariance by assuming that the finetuning of localization is signaled by the ratios of responses of adjacent units.

It is important to note that even a single-point stimulus activates more than one unit at a time. If this were not the case, or if a type of lateral inhibition were to operate such that only the maximally stimulated unit were to signal the brain, then localization would be no finer than the average spacing between adjacent units. Preserving the information about the relative responses of adjacent units permits very fine localization.

Let us now turn to the two-point discrimination task. If Stimuli 2 and 4 , each of amplitude .5 , were presented simultaneously in place of Stimulus 3 , then the response vector would be $(44+18,49+49,20+45$, $0+7)=(62,98,65,7)$ instead of $(70,100,70,0)$. It is apparent from this that the stimulus $2+4$ has nearly the same effect on the four units as does a single stimulus at an intermediate position and of 
twice the amplitude. Thus, if the two-point stimulus were presented just by itself, it is clear that it would be experienced as a single stimulus, though perhaps slightly "broader" than a single point. Now consider the stimulus consisting of Points 1 and 7, each of amplitude .5; here, the response vector would be $(50,35,35,50)$. This is a case where one or more units have response magnitudes less than more outlying units. According to Assumption (4), this stimulus configuration would give rise to the sensation of duality, provided that the decrement in response of the intervening units surpassed whatever the criterion value was. For intermediate separations of the two points, the model would predict sensory experience intermediate between that of "a single sharp point" and that of "two distinct points." This is precisely what is reported (Boring, 1921, 1942); one such set of reported perceptual categories with increasing separation is that attributed to Gates (Boring, 1942): point, circle, line, dumb-bell, two points.

If we were to consider an extended array of mechanoreceptive units like those in Figure 3, we could determine the spatial profile of their response magnitudes elicited by spatial-frequency stimulus gratings (with the amplitude of the stimulus being pressure, deformation, rate of deformation, or whatever stimulus property activates each unit). For very high frequencies, the integral of the product of the sine-wave stimulus and the receptive field sensitivity function would be the same for all units so that there would be no spatial modulation of the response profile. If the spatial frequency were gradually reduced, there would come a point where one unit would be differentially stimulated relative to its neighbor. Thus, there would be a spatial modulation ("ripple") of the profile of responses. If the grating were to move, so too would the ripple in the response profile, indicating that the mosaic of receptors taken together is phase-sensitive. The point being made is that such an array of mechanoreceptive units can be characterized as a low-pass spatial frequency filter, inasmuch as its response profile is modulated only by the lower spatial frequencies. Of course, the model was formulated to have this property so that it could explain the difference between resolution and localization. Incidentally, the model proposed here has a number of parallels with the explanation of how acute wavelength discrimination is accomplished with just a few photoreceptors of broad overlapping spectral sensitivities, which, when stimulated with wavelength mixtures, give rise to metamerism (the indistinguishability of spectral mixtures).

Although the model we have presented is in reasonable accord with the physiology of cutaneous mechanoreception (e.g., see Johansson, 1976;
Johansson \& Vallbo, 1976), it was not intended to be definitive. Rather, the purpose was merely to show that there is some physiological model which can account for the difference between resolution and localization using a common physiological pathway. However, even if the model were to prove correct with regard to the basic difference between localization and resolution, it is possible that the shift thresholds studied here owe their fineness to an additional mechanism. For example, the initial stimulus might cause a rapid adaptation of the junderlying mechanoreceptive units such that when the stimulus is moved, the resulting neural signal for location is equivalent to one representing a location even further displaced were there no adaptation. Yet another possibility would be that the motion per se is a trigger feature for motion-sensitive units having high directional selectivity.

Though the present result manifests the precision with which the tactile sense accomplishes the task of localizing stimulation, it cannot lay claim to being the first demonstration of what we have called tactile hyperacuity. Vierck and Jones (1969) presented evidence of rather fine size discrimination of objects impressed upon the arm; using plastic cylinders of varying diameters as stimuli and the method of forced choice, they obtained just noticeable size differences (measured in terms of diameter) which were roughly 10 times smaller than the two-point threshold for the same test location. Reasoning that the skin is primarily organized for localization and size discrimination, they argued for the rejection of "the two-point threshold as the standard measure of spatiotactile resolution" (p. 488). However, in light of the foregoing discussion, it should be evident that size discrimination in no way need depend on spatial resolution, for the pattern characteristics which mediate size discrimination will be largely invariant up to some extreme value of low-pass spatial filtering (blurring). Although spatial resolution by itself may have little consequence for a wide variety of spatial discrimination tasks, it nevertheless remains an important index of a system's spatial sensitivity.

\section{REFERENCE NOTE}

1. Collins, C. C., \& Madey, J. M. J. Tactile sensory replacement. Proceedings of the San Diego Biomedical Symposium, 1974.

\section{REFERENCES}

Apkarian-Stielau, P., \& Loomis, J. M. A comparison of tactile and tlurred visual form perception. Perception \& Psychophysics, 1975, 18, 362-368.

BERRY, R. N. Quantitative relations among vernier, real depth, and stereoscopic depth acuities. Joumal of Experimental Psychology, 1948, 38, 708-721.

Boring, E. G. The stimulus-error. American Journal of Psychology, 1921, 32, 449-471. 
Boring, E. G. The two-point limen and the error of localization. American Journal of Psychology, 1930, 42, 446-449.

Boring, E. G. Sensation and perception in the history of experimental psychology. New York: Appleton-Century-Crofts, 1942.

DisheR, D. R. The effect of pressure magnitude on cutaneous localization. Journal of General Psychology, 1933, 9. 390-404.

HeCHT, E. \& ZAJAC, A. Optics. Reading, Mass: Addison-Wesley, 1976.

HoAgland, H. A basis for cutaneous localization. Joumal of General Psychology, 1932, 6, 468-470.

JoHansSON, R. Skin mechanoreceptors in the human hand: Receptive field characteristics. In Y Zotterman (Ed.), Sensory functions of the skin in primates. New York: Pergamon Press, 1976.

Johansson. R., \& VALLBO, A. B. Skin mechanoreceptors in the human hand: An inference of some population properties. In Y. Zotterman (Ed.), Sensory functions of the skin in primates. New York: Pergamon Press, 1976.

Loomis, J. M. Tactile letter recognition under different modes of stimulus presentation. Perception \& Psychophysics, 1974, 16, 401-408.

Ludvigh, E. Direction sense of the eye. American Journal of Ophthalmology, 1953, 36, 139-143.

Pritchard, E. A. B. Cutaneous tactile localization. Brain, 1931, 54, 350-371.

Rivers, W. H. R., \& HeAd, H. A human experiment in nerve division. Brain, 1908, 31, 323-450.

Sinclair, D. Cutaneous sensation. New York: Oxford University Press, 1967.

VIERCK, C. J., JR., \& JoNEs, M. B. Size discrimination on the skin. Science, 1969, 63, 488-489.

VON BÉKÉsy, G. Sensory inhibition. Princeton, N.J: Princeton University Press, 1967.

WEINSTEIN, S. Intensive and extensive aspects of tactile sensitivity as a function of body part, sex, and laterality. In D. R. Kenshalo (Ed.), The skin senses. Springfield, Ill: Thomas, 1968.

WERNER, G., \& Whitsel, B. L. Functional organization of the somatosensory cortex. In A. Iggo (Ed.), Handbook of sensory physiology (Vol. II) Somatosensory system. New York: SpringerVerlag, 1973.

WESTHEIMER, G. Visual acuity and spatial modulation thresholds. In D. Jameson \& L. M. Hurvich (Eds.), Handbook of sensory physiology (Vol. 7/4) Visual psychophysics. Berlin: SpringerVerlag, 1972.

Westheimer, G. Spatial frequency and light-spread descriptions of visual acuity and hyperacuity. Journal of the Optical Society of America, 1977, 67, 207-212.
Westheimer, G., \& McKeE, S. Integration regions for visual hyperacuity. Vision Research, 1977, 17, 89-93. (a)

Westheimer, G.. \& MCKeE, S. Spatial configurations for visual hyperacuity. Vision Research, 1977, 17, 941-947. (b)

ZigLER, M. J. The experimental relation of the two-point limen to the error of localization. Journal of General Psychology, 1935, 13, 316-331.

\section{NOTE}

1. Although the two-point limen is taken as the conventional measure of static spatial resolution for the sense of touch, there are some problems inherent in its use. Strictly speaking, spatial resolution for a linear image processing system is the highest input spatial frequency (of maximim contrast) which gives a measurable response of the same frequency (Westheimer, 1977). Thus, for the sense of touch, the ideal stimuli for measuring static spatial resolution would be extended gratings which are sinusoidally modulated in amplitude in one spatial direction. Less ideal but still more suitable than the two-point stimulus is the configuration suggested by Westheimer (1977), which consists of two parallel bars, each 1 unit wide and 3 units long and separated by a 1-unit space. The problem with the two-point configuration is that spatial frequencies of periods greater than the two-point separation convey information about the target; put differently, the target is longer than it is wide so that even when the two points are not "resolvable," the impression might be of an elongated single target (Boring, 1921). In optics, resolution has been measured using two-point configurations; resolution is defined as that separation which satisfies the Rayleigh criterion (roughly, there is slight dip between the two peaks in the image diffraction pattern). The corresponding criterion for tactile resolution would be that separation which just gives rise to the impression of duality; other, less stringent criteria would tend to credit the tactile sense with better resolution than it possesses. In practice, however, the two-point configuration probably suffices as a measure of acuity (reciprocal of the upper limiting spatial frequency).

(Received for publication February 20, 1978; revision accepted October 3, 1978.) 